\title{
Delayed Splenic Rupture: A Case Report and Literature Review
}

\author{
Alaa Sedik ${ }^{\mathrm{a}, \mathrm{b}}$, Fawaz AlRashed ${ }^{\mathrm{a}}$, Tarek AlDawoodi ${ }^{\mathrm{a}}$, Mohamed ElSayed ${ }^{\mathrm{a}}$, \\ Salwa Elhoushy ${ }^{a}$
}

\begin{abstract}
The spleen is one of the most commonly injured organs in the body after blunt abdominal trauma. Our objective was to present a case of the rare entity called "delayed splenic rupture", which is the delayed manifestation of splenic injury following a trauma in which the initial ultrasonography study was normal. We also review the previous 13 cases up to 2015 and offer our recommendations. We present a case of a 60 -year-old male with a history of road traffic accident 3.5 days prior to admission to our hospital with initial normal ultrasonography scan done at a peripheral referring hospital. He was evaluated and proved to have a large subcapsular splenic hematoma and significant hemoperitoneum. He was offered splenectomy.
\end{abstract}

Keywords: Spleen rupture; Blunt; Abdominal trauma

\section{Introduction}

Delayed rupture of the spleen (DSR) was first described by Baudet in 1902 [1]. He suggested that the period of time from the injury to the splenic rupture was $48 \mathrm{~h}$ or more and is characterized by a period of clinical quiescence, now referred to as "the latent period of Baudet" [2]. Using new diagnostic techniques in the 1970s, the incidence was re-evaluated and considered to be around $1 \%$. This took into account cases which represented delayed diagnosis of splenic injury. The importance of DSR lies in the relatively high mortality rates $(5-15 \%)$, compared to the $1 \%$ mortality associated with acute splenic injury [2].

\section{Case Report}

A 60-year-old Saudi male known to have insulin-dependent diabetes mellitus and hypertension on regular medications was admitted to our emergency room as a referral from a nearby

Manuscript submitted April 05, 2017, accepted May 02, 2017

aSurgery and Medical Department, King Khalid Hospital Hail, Saudi Arabia ${ }^{b}$ Corresponding Author: Alaa Sedik, King Khalid Hospital Hail, KSA.

Email: asedik59@yahoo.com

doi: https://doi.org/10.14740/jcs323w peripheral hospital. He was admitted as a road traffic accident 3.5 days ago and put on observation. They transferred him for opinion of thoracic surgery and neurosurgery as he had multiple left lower rib fractures and back pain. His lab works together with initial and repeated focused assessment with sonography for trauma (FAST) in the referring hospital were within normal. On arrival, he was conscious, alert, oriented with blood pressure 130/70 $\mathrm{mm} \mathrm{Hg}$, pulse 140/min and normal oxygen saturation. There was pallor. He experienced recent left upper quadrant abdominal worsening pain for few hours prior to presentation to our hospital. Clinically, there was tenderness over lower left chest and left upper abdominal quadrant but normal air entry bilateral and no rigidity or rebound. Patient was resuscitated according to Advanced Trauma Life Support protocol. Initial blood works showed $\mathrm{Hgb}$ of $12 \mathrm{gm} \%$ and serum creatinine of 254 units/L and blood urea nitrogen was raised. Coagulation profile showed international normalized ratio of 1.9 and prothrombin time and partial thromboplastin time were elevated also. Arrangement of fresh frozen plasma (FFP), platelets, and packed red blood cells (PRBCs) was made.

Transfusion of FFP was started immediately. Anteroposterior (AP) chest X-ray showed multiple left 6-9 rib fractures and raised left copula of diaphragm with no hemo- or pneumothorax. AP X-ray of the pelvis was normal. FAST ultrasonography showed moderate free intraperitoneal fluid around the liver, spleen and in the pelvis. Echocardiography was done and was normal. Nephrology consultation was done; then an urgent contrast-enhanced computerized scan (CT) of abdomen was done and showed a huge subcapsular splenic hematoma with evidence of a recent bleed inside with moderate fluid collection (Fig. 1). Fractures of 5-9 ribs and a small left hemo-pneumothorax were noted. CT of brain showed small tempro-parietal contusions. CT of spine and pelvis was normal. The patient was diagnosed as a delayed rupture of large subcapsular splenic hematoma. He was seen by neurosurgeon and thoracic surgery. The situation was discussed and a consent for laparotomy was obtained. Fresh blood $2.5 \mathrm{~L}$ was seen and 4 quadrant packing was done as blood pressure dropped to $80 / 50 \mathrm{~mm} \mathrm{Hg}$. Aggressive resuscitation was done with fluids, PRBCs, FFP, and platelets, and then blood pressure came to $110 / 60 \mathrm{~mm} \mathrm{Hg}$.

The left quadrant pack was removed and fresh blood was found to come from the area. Huge clots were removed and rapid splenectomy was performed (Fig. 2). The tail of pancreas was damaged and debrided also. All other packs were removed. A bleeding grade II $(6 \mathrm{~cm})$ right hepatic laceration on the diaphragmatic surface was seen and was treated with lo- 

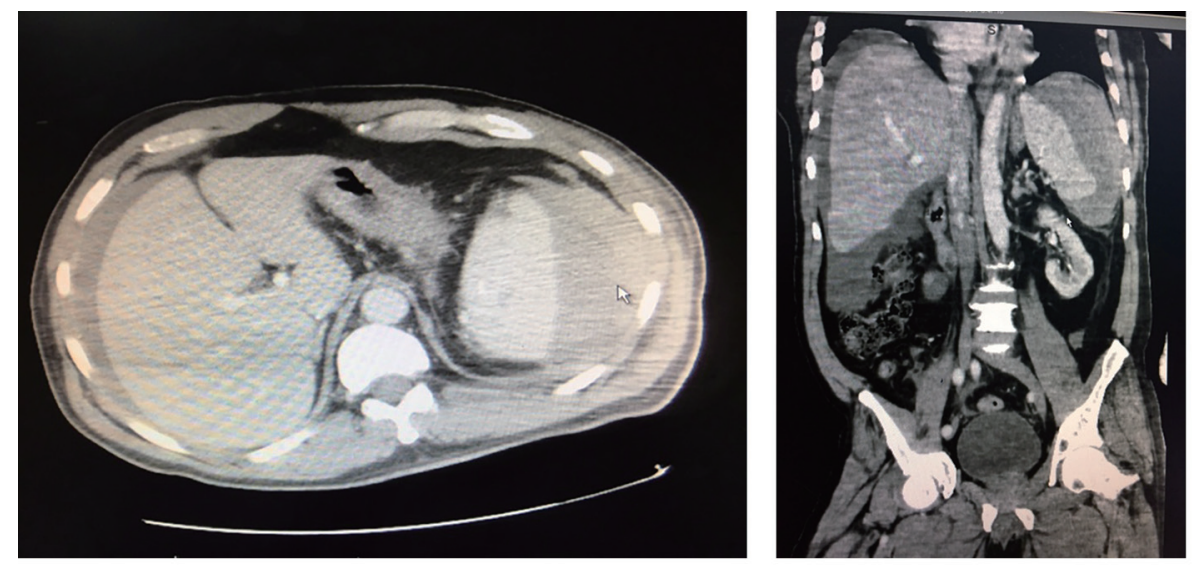

Figure 1. Large subcapsular splenic hematoma in a grossly normal spleen, together with significant hemoperitoneum.

cal hemostats. There was a large contusion and sero-muscular tears of the colon and small bowel of transverse mesocolon. No other gross serious injuries were seen except a non-pulsating, non-expanding left perinephric hematoma. There was a significant oozing from the laparotomy wound, splenic bed, also the liver laceration. No determined source of the bleeding was made. Decision was made to terminate surgery and packs were left. Skin was closed after two large intra-peritoneal drains. He received 4 units PRBCs, FFP, and platelets perioperatively. The patient was admitted to ICU. A left-sided inter-costal tube was inserted on the first postoperative day for a pneumothorax. A second look laparotomy was done after $48 \mathrm{~h}$ as coagulopathy and renal functions improved and patient became more stabilized. All packs were removed with no active bleeding. Saline lavage was done and mass closure technique was followed to close the abdomen. He was extubated on the third day and shifted to step down unit on the next day. He was confused and irritable. He started entral feeding and tolerated it well with normal motions. The drainage fluid accounted for $300-400$ $\mathrm{mL} / 24 \mathrm{~h}$ and was serous. Sample was sent for biochemistry. On the sixth day, blood works showed evidence of pancreatitis with serum amylase 1,200 and lipase 4,000 units/L. Gastroenterology diagnosed the case as traumatic pancreatitis and offered him conservative treatment. A contrast-enhanced abdominal CT was done and showed no gross pathology. Brain CT and MRI were advised by neurologist and showed no recent pathology. Drainage fluid was negative for amylase. Patient responded well to treatment and resumed oral feeding. The drainage fluid decreased then tubes were removed on 12th day. Serum amylase and lipase levels came to normal. Skin sutures were removed on the 15 th day. Patient was discharged and was doing fine on follow-up in outpatient clinic.

\section{Discussion}

The mechanism of DSR is not known. There are two predominant theories in the literature.

The first theory describes a perisplenic hematoma that has been temporarily sealed off by surrounding organs and structures like omentum, only to rupture freely into the peritoneal cavity at a later time. The second, more widely accepted theory proposes increasing subcapsular tension, most probably from clot lysis and increasing oncotic pressure, as a cause of delayed rupture $[3,4]$.

DSR was defined by Benjamin as rupture of spleen more than $48 \mathrm{~h}$ after trauma. Delayed rupture is seen in less than $10 \%$ of cases. The time period is based on the "latent period of Baudet" of $48 \mathrm{~h}$ which Baudet described as time from injury to rupture $[3,5,6]$. The first case of DSR documented by CT was reported in 1981 by Toombs et al [7]. Weinberg et al recommended serial CT scans in all cases of NOM to identify latent formation of splenic artery pseudo-aneurysms. While indications for repeat CT in splenic trauma are limited, DSR should always be considered a possibility in hemodynamically unstable patients [8].
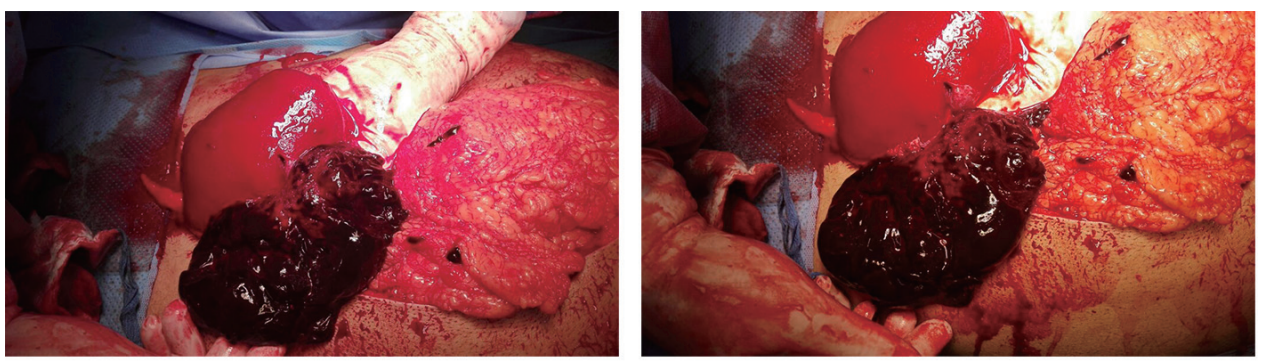

Figure 2. Intraoperative findings: large clot shown with grossly normal looking spleen with pieces of its torn capsule. The hand of the surgeon shown to control the hilum as there was active bleeding from the tear. 
Based upon his experience with a single patient, MacIndoe in 1932, proclaimed a $10-15 \%$ incidence of delayed splenic bleeding in patients in whom an early diagnosis splenic injury was not made. In blunt abdominal trauma, the spleen is one of the most commonly injured organs. The splenic rupture may be acute, delayed or occult in nature. Acute rupture, characterized by intraperitoneal bleeding and features of shock, occurs in more than $90 \%$ of cases. The entity of DSR is often challenged $[8,9]$.

However, ultrasonography performs poorly for low-grade splenic injury (American Association for the Surgery of Trauma scale I or II). It is most sensitive to detect grade III or above splenic injuries based on the presence of hemoperitoneum. In case of doubt, CT remains the gold standard for diagnosing and precisely assessing the severity of splenic injury, provided that the patient is stable $[10,11]$.

\section{Conclusion}

Obtaining a detailed history about the mechanism of injury, careful clinical assessment, high index of suspicion and liberal use of imaging have been suggested to minimize the risk of "delayed recognition" of splenic injury. Bedsides, ultrasonography emerges as an easily available, highly sensitive and specific, fast and non-invasive method for emergency physicians to detect major intra-abdominal organ injuries. CT confirms and grade the injury in most cases. A normal appearing spleen on initial imaging should not sway one from considering DSR as a potential delayed complication of a traumatic event.

\section{Acknowledgments}

We are so grateful to Osama Sedik for help in preparing the figures.

\section{Financial Support}

None.

\section{Conflicts of Interest}

There are no conflicts of interest.

\section{References}

1. Baudet R. Ruptures de la rate. Medicine Practique. 1907;3:565.

2. Schwartz, Shires and Spencer. Principles of Surgery. 5th edition, McGraw-Hill Book Company, 1989.

3. Farhat GA, Abdu RA, Vanek VW. Delayed splenic rupture: real or imaginary? Am Surg. 1992;58(6):340-345.

4. Maingot's Abdominal Operations. In: Schwartz SI, Ellis H, Husser WC, editors. 9th edn. 1990. Vol II. pp. 1677.

5. Kluger Y, Paul DB, Raves JJ, Fonda M, Young JC, Townsend RN, Diamond DL. Delayed rupture of the spleen - myths, facts, and their importance: case reports and literature review. J Trauma. 1994;36(4):568-571.

6. Coughlin F, Aanning HL. Delayed presentation of splenic trauma following colonoscopy. S D J Med. 1997;50(9):325-326.

7. Toombs BD, Lester RG, Ben-Menachem Y, Sandler CM. Computed tomography in blunt trauma. Radiol Clin North Am. 1981;19(1):17-35.

8. Weinberg JA, Magnotti LJ, Croce MA, Edwards NM, Fabian TC. The utility of serial computed tomography imaging of blunt splenic injury: still worth a second look? J Trauma. 2007;62(5):1143-1147; discussion 1147-1148.

9. Fernandes CM. Splenic rupture manifesting two years after diagnosis of injury. Acad Emerg Med. 1996;3(10):946947.

10. Peitzman AB, Makaroun MS, Slasky BS, Ritter P. Prospective study of computed tomography in initial management of blunt abdominal trauma. J Trauma. 1986;26(7):585-592.

11. Richards JR, McGahan JP, Jones CD, Zhan S, Gerscovich EO. Ultrasound detection of blunt splenic injury. Injury. 2001;32(2):95-103. 